\title{
UCRL-CONF-212639
}

LAW RENCE LIVERMORE N A T IO N A L LABORATORY

\section{UV PRE-IONIZED RAIL-GAP SWITCH FOR STACKED BLUMLEIN PULSE GENERATORS*}

M. A. Rhodes

June 1, 2005

IEEE International Pulsed Power Conference Monterey, CA, United States June 14, 2005 through June 17, 2005 
This document was prepared as an account of work sponsored by an agency of the United States Government. Neither the United States Government nor the University of California nor any of their employees, makes any warranty, express or implied, or assumes any legal liability or responsibility for the accuracy, completeness, or usefulness of any information, apparatus, product, or process disclosed, or represents that its use would not infringe privately owned rights. Reference herein to any specific commercial product, process, or service by trade name, trademark, manufacturer, or otherwise, does not necessarily constitute or imply its endorsement, recommendation, or favoring by the United States Government or the University of California. The views and opinions of authors expressed herein do not necessarily state or reflect those of the United States Government or the University of California, and shall not be used for advertising or product endorsement purposes. 


\title{
UV PRE-IONIZED RAIL-GAP SWITCH FOR STACKED BLUMLEIN PULSE GENERATORS *
}

\author{
Mark A. Rhodes \\ Lawrence Livermore National laboratory, PO Box 808, L-485 \\ Livermore, CA, USA
}

\begin{abstract}
Stacked Blumlein Pulse Generators comprised of parallel-plate transmission lines are potentially a useful pulse-power architecture for high-gradient, compact, electron-beam accelerators and other applications. Such pulse generators require a low-inductance, fast $(<5 n)$ switch per stage to erect the stack and produce the desired output pulse. We are developing a rail-gap switch tightly integrated with the stack for this application. We employ ultraviolet light (UV) to pre-ionize the switch, which facilitates prompt, low-jitter, and potentially multichannel operation. A novel aspect of our switch is that the source of the UV is a conventional Xenon flashlamp. This allows variation of the switch pressure and gas without affecting the flashlamp operation. We can operate our switch in either triggered or self-breaking mode. Here we present initial results of a two-stage, stacked Blumlein operating in self-break mode. We compare the switch performance to gas-switch scaling laws with respect to resistive-phase risetime and trigger delay as a function of gas density, gap-length, and gap-voltage.
\end{abstract}

\section{I.INTRODUCTION}

We are developing a high-voltage pulse generator technology based on charged-line, parallel-plate, Blumlein transmission lines. Parallel-plate transmission lines are particularly well suited for stacking to achieve much higher output voltages than we can achieve with single stages. Such Stacked Blumlein (SBL) pulse generators have a wide range of pulsed power applications including particle accelerators and EMP generators.

The output pulse shape of an SBL is ideally a rectangular pulse when coupled to a matched load. However, in practice the actual pulse shape depends on the actual properties of the transmission line structure, actual properties of the load, and perhaps most importantly, the characteristics of the switch used to discharge each stage. All charged-line pulse generators require such a switch and in many cases this switch is the main limiting factor in performance. A wide variety of switches are possible. At lower voltage levels, semiconductor switches and optical switches are viable. Since we are interested high-voltage per stage (up to $100 \mathrm{kV}$ ) and line impedances that require kiloamp level switch currents, pressurized spark-gap switches remain one of the most viable alternatives.

\section{II.SWITCH DESIGN}

When designing a pulse generator with rise-times approaching 1ns, it is important to consider the design of the switch and the transmission line in an integrated way. To design our switch, we considered two widely known spark-gap scaling laws. The scaling law for a spark gap resistive-phase rise-time is given by[1]

$$
t_{r}(n s)=\frac{1894}{(N Z)^{1 / 3} E^{4 / 3}}\left(\frac{\rho}{\rho_{0}}\right)^{.5}
$$

where $\mathrm{N}$ is the number of switch channels, $\mathrm{Z}$ is the line impedance in ohms, $\mathrm{E}$ is the electric field in $\mathrm{kV} / \mathrm{cm}, \rho$ is the gas density of the switch operating gas, and $\rho_{0}$ is the gas density of air at STP. We require a low-jitter switch so our design will scale to larger systems with many more stages. To achieve low jitter, the switch delay time (time after trigger or time after breakdown voltage is exceeded) must not be too much longer than the required switch risetime. We consider the scaling law for switch delay time[2]

$$
t_{h}(n s)=9.78 \times 10^{13} \frac{\rho^{2.44}}{E^{3.44}}
$$

We solve these two scaling laws for $\mathrm{E}$ and obtain an expression for the required gas density as a function of switch rise-time and switch delay.

$$
\rho=\left[\frac{2.437 \times 10^{-2} t_{h}^{291}}{\left((N Z)^{1 / 3} \rho_{0} t_{r}\right)^{3 / 4}}\right]^{2.985}
$$

Then we go back to Eq. (1) or Eq. (2) and solve for the electric field. If we choose a voltage, then we also have chosen the gap and the switch design is complete with regard to gap, voltage and operating pressure for a given

\footnotetext{
* This work was performed under the auspices of the U.S. Department of Energy by the University of California, Lawrence Livermore National Laboratory under contract No. W-7405-Eng-48
} 
gas. We have used this solution of the gas switch scaling laws to examine various aspects of switch design.

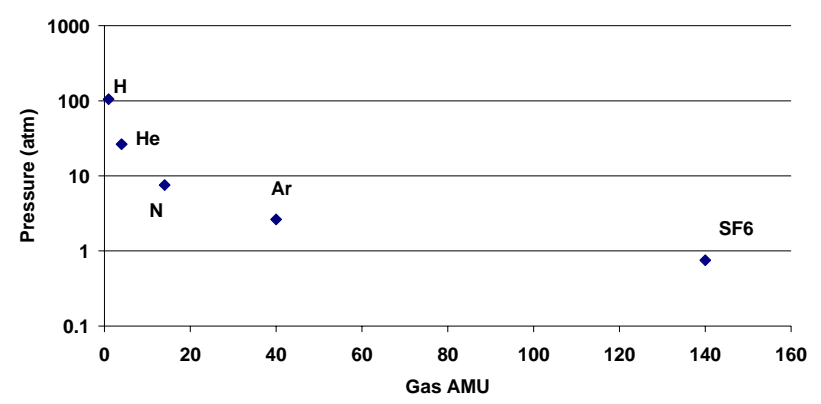

Figure1. Required pressure as a function of gas AMU for fixed switch parameters.

In Figure 1, we show the required gas pressure of some common switch gases for a fixed set of line and switch parameters ( $\left.\mathrm{t}_{\mathrm{r}}=5 \mathrm{~ns}, \mathrm{t}_{\mathrm{h}}=200 \mathrm{~ns}, \mathrm{Z}=13 \mathrm{ohms}\right)$ and we assume 10 channels. We see that heavier gases allow lower, more practical operating pressures. We chose $\mathrm{SF}_{6}$ as our baseline gas for this reason.

We can also see how risetime affects switch design.

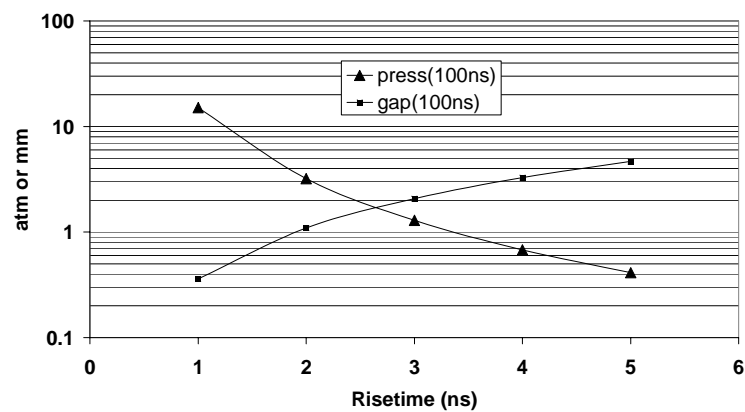

Figure 2. Switch gap and pressure as a function of desired risetime for fixed switch delay time.

We see in Fig. 2 that for faster risetime, we must have higher pressure and decreased gap-size. We can define a "zone of practicality" where switch pressure is 1-10 atm and switch gap is $1-10 \mathrm{~mm}$. Gaps less than $1 \mathrm{~mm}$ would be hard to realize with good accuracy over many switches and pressures less than 10 atm. are obviously more practical.

\section{III.UV ILUMINATION}

It is well known that UV illumination of spark-gap switches improves jitter performance [3]. For our switch design we have chosen conventional Xenon flashlamps as the source of UV. Use of flashlamps is well suited to our geometry. We can place the lamps close to the switch electrodes and the lamps have their own sealed gas environment. Changes in the switch gas or pressure therefore do not affect the lamp operation. Lamps are much less expensive and simpler to implement than a UV laser. We believe this may be the first time where flashlamps have been used to facilitate spark-gap operation.

\section{EXPERIMENTAL SETUP}

A side-view diagram of the experimental setup is shown in Fig. 3. A CAD model of the experiment is shown in Fig. 4. A closer view CAD model of the switch end is shown in Fig. 5 and a photograph of the fielded experiment is shown in Fig. 6.

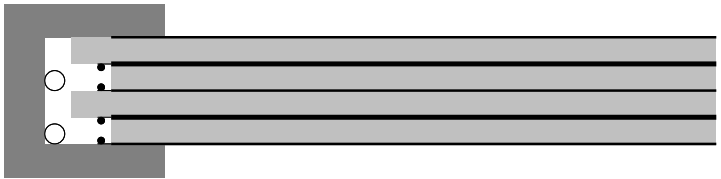

Figure 3. A side view diagram of our two-stage Stacked Blumlein pulse generator.

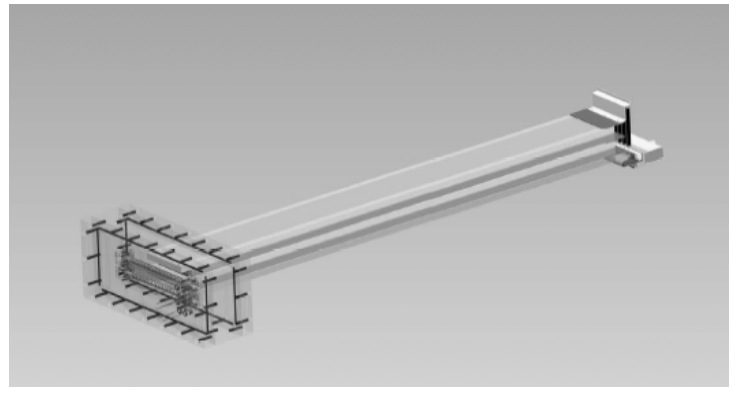

Figure4. A full CAD model of the experimental setup.

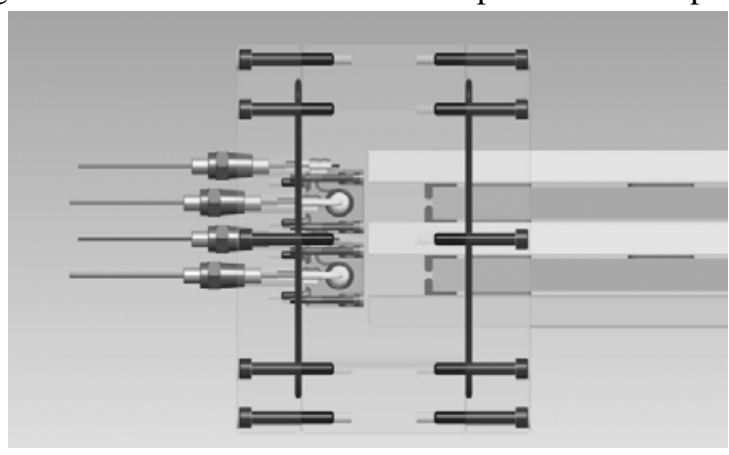

Figure 5. A CAD model showing a closer view of the gas box, switch electrodes, and flashlamps.

The stack is comprised of four layers of Duroid ${ }^{\circledR}$ [4] material with an $\varepsilon_{\mathrm{r}}$ of 10.2. The length of the line is $120 \mathrm{~cm}$ yielding an expected pulse width of 26ns. The width of the line is $10 \mathrm{~cm}$ and the line thickness is 1.27 cm yielding an expected impedance of 14.75 ohms per layer or 59 ohms for the output impedance of the twostage stack. The Duroid material is clad with 2oz copper and we used an oven process with conventional solder to attach the layers together both electrically and mechanically. The switch end is enclosed in a polycarbonate box to allow the switch to operate with different gas and pressure than the rest of the stack. A polycarbonate flange is epoxy-potted to the stack and 
forms the pressure seal. The back cover of the gas box provides for mounting of the flashlamps along with electrical feedthroughs for the flashlamps and their triggers. The other end is the load end where we terminate the line with a matched resistive load.

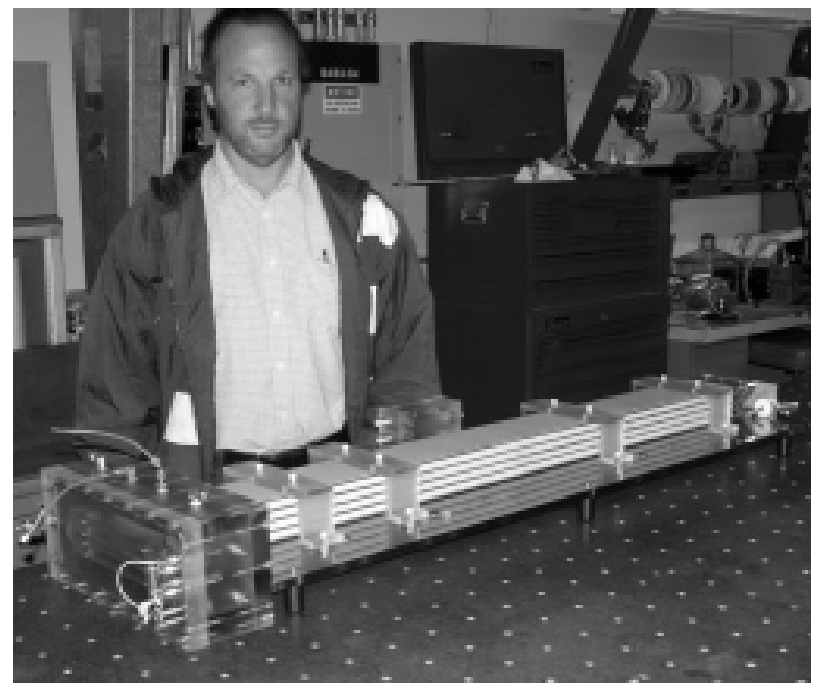

Figure 6. A photograph of the completed two-stage SBL with flashlamp illuminated spark gap switches.

We designed the switch electrodes to press in between the Duroid layers. This provided us with the flexibility to easily change electrodes allowing changes in gap, electrode shape, or electrode material.

To diagnose the operation of the stack, we incorporated three voltage probes: one across each switch and one across the load. These voltages probes are comprised of $1000-O h m$ resistors placed across the measurement nodes. We use a wide-band current transformer to measure the current in the probe resistor. This signal is proportional to the voltage across the probe and DC-isolated. We record these three signals on a waveform digitizer.

The stack is charged with a pulse-charge system. This system feeds the two-stage stack through a balanced network of inductors. The balanced charging network charges both stages synchronously, which is important for self-breaking switches. We are able to vary the charging speed by changing the inductor set. In practice, we varied the charging speed from 500ns down to as fast as $100 \mathrm{~ns}$.

The Xenon flashlamps are driven with a capacitor discharge supply. The current pulse is approximately $10 \mu \mathrm{s}$. We trigger the flashlamps in advance of the pulse charge and set the delays so the stack fires near the peak of the flashlamp current pulse. On the time scale of the pulse-charging and stack firing, the flashlamp current is essentially constant

To design the final switch electrodes, we choose target switch parameters of $t_{r}=2 n s$ and $t_{h}=30 n s$. This yields a set of gaps for operation at any desired voltage. The data presented here was taken with a $1.6 \mathrm{~mm}$ gap $(20 \mathrm{kV}$ nominal switching voltage at 1 -atm. of $\mathrm{SF}_{6}$. In principle, this stack is capable of much higher voltage per stage by installing larger gaps but the electrical week point is where the pressure flange is potted to the stack. We kept the switching below $30 \mathrm{kV}$ to preserve the life of the stack.

\section{V.EXPERIMENTAL RESULTS}

We have operated our SBL with two electrode widths (full line width of $10 \mathrm{~cm}$ and a short electrode $6.4 \mathrm{~mm}$ wide). We have experimented with different electrode profiles and have run with two gases (pure $\mathrm{SF}_{6}$ and $15 \%$ Argon $85 \% \mathrm{SF}_{6}$ ) at pressures from 0-psig to 25-psig. Generally, we achieved the best results with the Argon gas mix combined with a sharpened-edge electrode on the anode side of the switch.

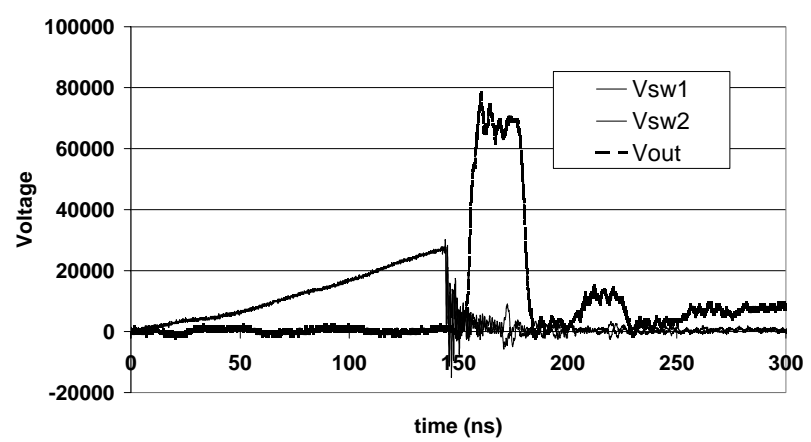

Figure 7. Typical data set showing pulse charging, switching, and output pulse.

In Figs. $7 \& 8$ we show a typical set of raw data. Voltage monitors across each switch $\mathrm{V}_{\mathrm{sw} 1}$ and $\mathrm{V}_{\mathrm{sw} 2}$ show the full pulse charge event and subsequent self-break switching. The waveforms from the two stages are directly on top of each other. The $70 \mathrm{kV}$ output pulse is also shown and appears $13 \mathrm{~ns}$ after switching (one-way transit time). The $10 \mathrm{kV}$ reflected pulse indicates that the load did not exactly match the stack impedance.

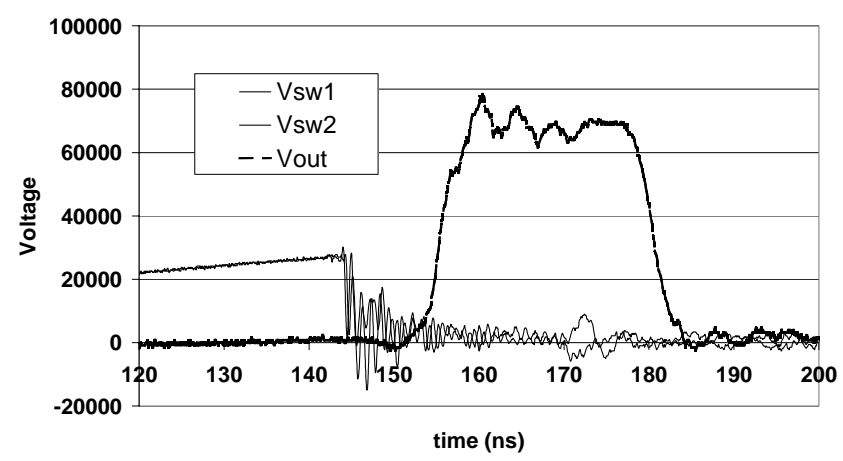

Figure 8. A zoomed in view of the same typical data set shown in Fig 7. 


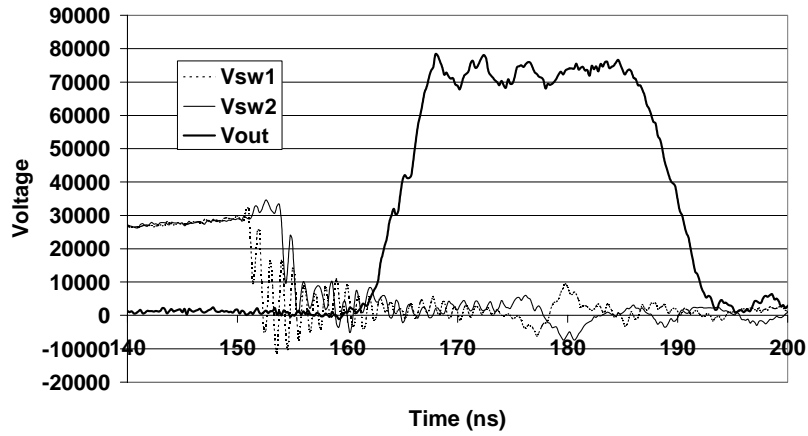

Figure 9. A dataset where the two stages fired about 2.5ns apart.

In Figure 9, we show a shot with the same conditions where the two stages fired about $2.5 \mathrm{~ns}$ out of synchronization. This leads to a "smearing out of the output pulse rise time.

We mentioned previously that we varied electrode shape, gas mix, and flashlamp current. We found the best results with the $15 \%$ Argon mix and a sharpened electrode on the positive (anode) side of the switch. We considered switching-promptness, shot-to-shot jitter, and shot-to-shot synchronization between stages as metrics. The electrode shape and gas mix only effect the very beginning of the switching event. In Figs. 7-9, the voltage at the switch is seen to increase monotonically as it follows the pulsecharge waveform and then suddenly collapses. In the less than ideal configurations, we observe that the voltage across the switch clamps for 1-10ns before switching occurs. We identify this as a "cooking phase" where current has started to flow but the voltage has not collapsed. This situation is clearly evident in Fig. 10.

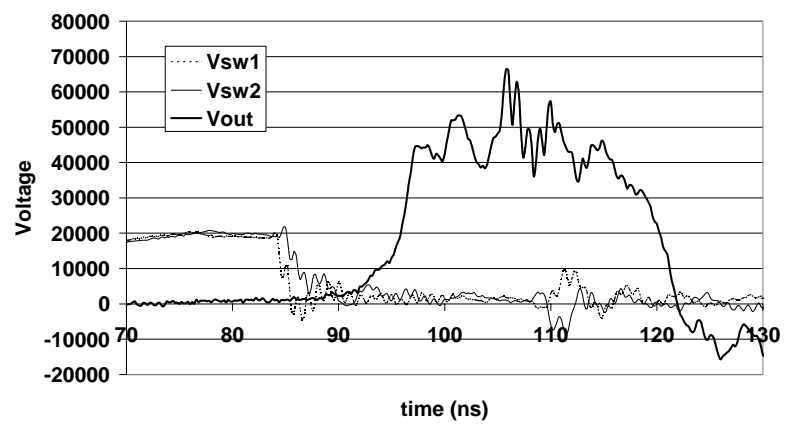

Figure 10. Sharp cathode electrode leads to non-prompt switching.

We found using a sharp electrode on the cathode side of the switch caused the longest "cooking times". This leads to a foot at the beginning of the output pulse.

At the fastest pulse-charge speed, we observed multichannel operation using the wide rails. We compared the output pulse to other shots where we used the narrow rails forcing single channel operation. We did not see any difference in the resulting output pulse. We expected the wide rails to provide lower inductance operation and hence, faster risetime. This remains an open issue for further research.

\section{VI.CONCLUSION}

We have presented a design methodology for highvoltage gas switches based on widely accepted empirical scaling laws. We have used this methodology to design a switch for planar transmission line, stacked Blumlein pulse generators. We have built and tested a two stage SBL. We have also demonstrated the use of Xenon flashlamps as a source of UV to allow synchronization of self-breaking switches with low jitter without expensive or complicated trigger systems. Our pulse generator works as expected generating a 26ns pulse with a risetime in good agreement with the predicted $t_{r}$.

\section{ACKNOWLEDGMENTS}

Many other people helped in the design, construction and operation of this experiment. We would like to specifically acknowledge Fred Allen for doing all the CAD design work, Wayne Jensen for the oven soldering and plastics work, Ed Gower and Joel Stanley for their assistance in the lab, Barry Smith at Advanced Photon Technology for developing the flashlamp, and Richard Miller at Titan Pulse Sciences for his discussions about gas switching.

\section{REFERENCES}

[1] J.C. Martin, "Solid, Liquid, and gaseous Switches," Texas Tech University Pulse Power Lecture Series, No. 30, 1981.

[2] T.H. Martin, "An Empirical Law for Gas Switch Breakdown Delay," in $7^{\text {th }}$ Pulsed Power Conference, 1989, pp. 73-79.

[3] T. Nitta, N. Yamada, and Y. Fujiwara, "Area Effect of Electrical Breakdown in Compressed $\mathrm{SF}_{6}$," IEEE Trans. Power Apparatus and Systems, vol. PAS-93, No. 2, pp. 623-9, 1974.

[4] Duroid is a registered trademark of the Rogers Corporation, Chandler, AZ. 